\title{
3D Printing of Artificial Breast Mould Based on the Real Medical Images
}

\author{
Dariusz Mikołajewski ${ }^{1}$, Piotr Prokopowicz ${ }^{1, *}$, Zbigniew Szczepański ${ }^{1}$, Joanna Nowak ${ }^{1}$, Marek \\ Macko ${ }^{1}$ and Emilia Mikołajewska ${ }^{2}$
}

${ }^{1}$ Institute of Mechanics and Applied Computer Science, Kazimierz Wielki University, Chodkiewicza 30, 85-064 Bydgoszcz, Poland

${ }^{2}$ Department of Physiotherapy, Ludwik Rydygier Collegium Medicum, Nicolaus Copernicus University, 3 Maja 6, 85-016 Bydgoszcz

\begin{abstract}
Three-dimensional (3D) printing and reverse engineering are usually superior to traditional teachnologies due to patient-tailored approach, scalability, and lower costs. This paper aims at the presentation of the own concept of the use of 3D medical images and reverse engineering for breast reconstruction for education and everyday clinical practice purposes. Presented concept of 3D breast reconstruction constitutes a relatively novel solution, and its further development may lead to the novel family of of patient-tailored solutions and optimized 3D-based technology seful in everyday clinical practice and online e-learning system.
\end{abstract}

Keywords: 3D printing; reverse engineering; medical images; implants; anatomy education.

\section{Introduction}

Reconstructive breast surgery aims at realistic, aesthetic, and symmetrical breasts, but required reconstructed breast's symmetry and appearance usually needs for multiple complex procedures [1]. 3D surface imaging technology allow for an accurate, reliable, and simple breast volumetric analysis, but it is not cheap, thus alternative/adjunct technologies (web-based 3D surface imaging, 4D imaging, and 3 D printing) appear promising $[2,3]$.

Three-dimensional (3D) printing (additive manufacturing) uses various materials and technologies (including poly-materal printing) to produce 3D objects, usually layer-by-layer. 3D printing and reverse engineering are usually superior to traditional teachnologies due to patient-tailored approach, scalability, and lower costs. Moreover traditional design and manufacturing for everyday clinical practice purposes have severe limitations: slow preparation and usually weak personalization of products [4-7].

Reverse engineering allows for digitizing real objects (body parts) for replication or modification (e.g. individualization, use of the other material) purposes.

$3 \mathrm{D}$ printing and reverse enginnering is used in everyday clinical practice in following areas:

- customization of medical products surgery, drug fabrication, and rehabilitation engineering purposes,

- education,

modeling and testing of implants (bones, soft tissues, whole organs) and assistive technology,

- pre-operation procedures (face/head/whole body scanning, 3D printing of patient-specific models).

$3 \mathrm{D}$ printing and reverse engineering may be combined with existing or novel therapeutic methods and techniques. It can significantly shorten track between 
diagnosis and related equipment, implants (artificial, biologic, hybrid), regenerative scaffolds, and cell-specific replacement tissue and organs [49].

This paper aims at the presentation of the own concept of the use of 3D medical images and reverse engineering for breast reconstruction for education and everyday clinical practice purposes.

\section{Experimental Section}

The title of our project is "Concept of an innovative method of artificial organs production based on anatomic features of the patient". Essential part of the project are novel (3D-based) approaches toward imitating physical and mechanical properties of tissues and organs based on their 3D-scans, computed tomography (CT) and magnetic resonance imaging (MRI). Used reverse engineering technology enables to gather, storage, modificate, re-design, and manufacture of relatively complex objects from its original structures or digitized images (figure 1). Particular area of application is breast reconstruction based on symmetric image of the healthy breast.

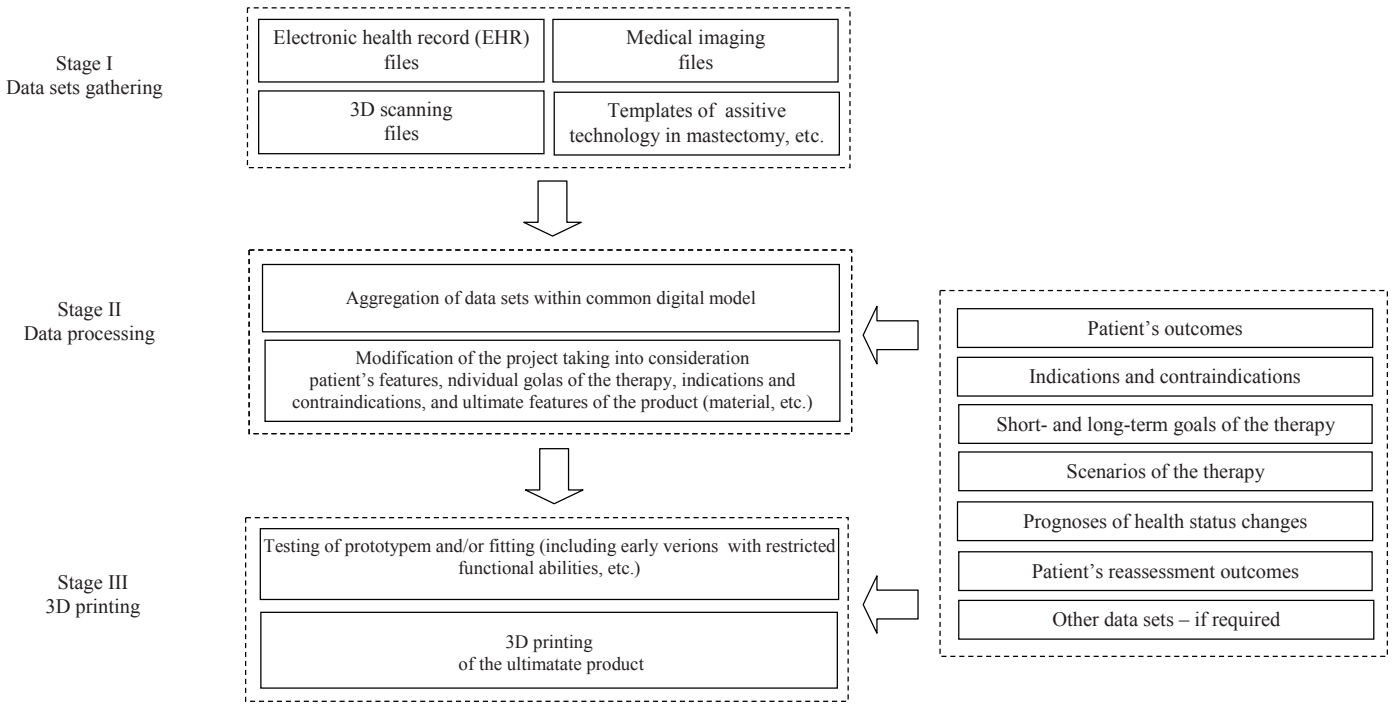

Fig. 1: Concept of the breast prostheses fabrication process [4-7].

\subsection{Results}

\section{Results and Discussion}

The current concept of 3D breast reconstruction offers many possibilities for both scientists and clinicians and may be easily developed toward standardized solution (figures 2-4). Process is relatively low cost, an its outcomes are promising. Limited conversion of 2D images to 3D is possible. Concept may be regarded novel and supplementary, but it is still at the begnning of its development toward 3D printing the fully clinically functional solutions.

There is possibility to:

$\checkmark$ convert standard imaging data into a CAD file,

$\checkmark$ use various $3 D$ reconstruction software,

$\checkmark$ fabricate 3D models using various 3D printing techniques.

Common belief is our approach meets possibilities, quality, and quantity comparable with similar solutions described in the scientific and clinical literature. Development of the concept can constitute another milestone toward on-demand printed customized biological tissues/organs/ implants.

\subsection{Discussion}

Preposed solution is regarded supplementary but it can fill existing gap within traditional approach to patients after mastectomy. Aforementioned technologies allow for:

$\checkmark$ early (before surgical intervention) planning of assistive technology,

$\checkmark$ digital quantification of breast tissue, despite its complexity,

$\checkmark$ simple and quick preoperative planning,

$\checkmark$ symmetric breast reconstruction,

$\checkmark$ increased efficiency, 
(a)

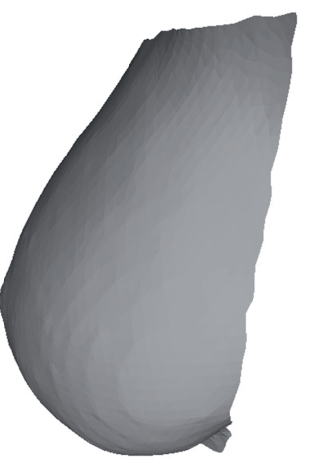

(c)

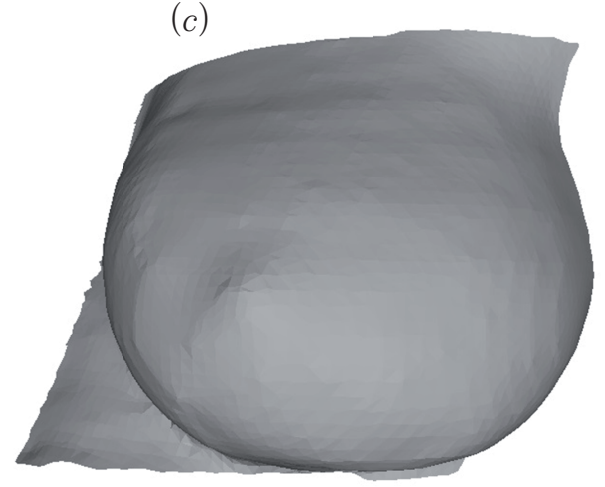

Fig. 2: Stage l of printing of breast based on its 3D scan: data sets gathering [4-7].
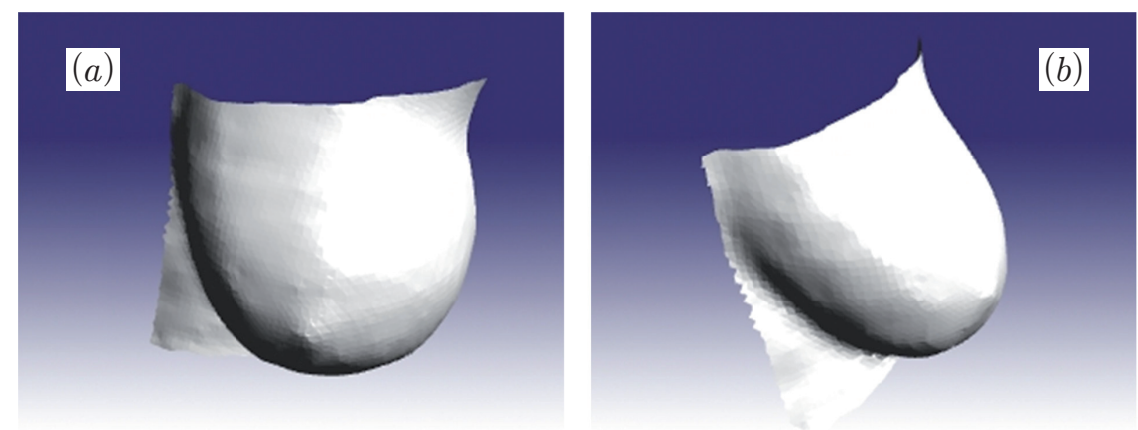

$(c)$

Fig. 3: Stage ll of printing of breast based on its 3D scan: data processing [4-7].

18 VOLUME 21, №. 4, 2017 

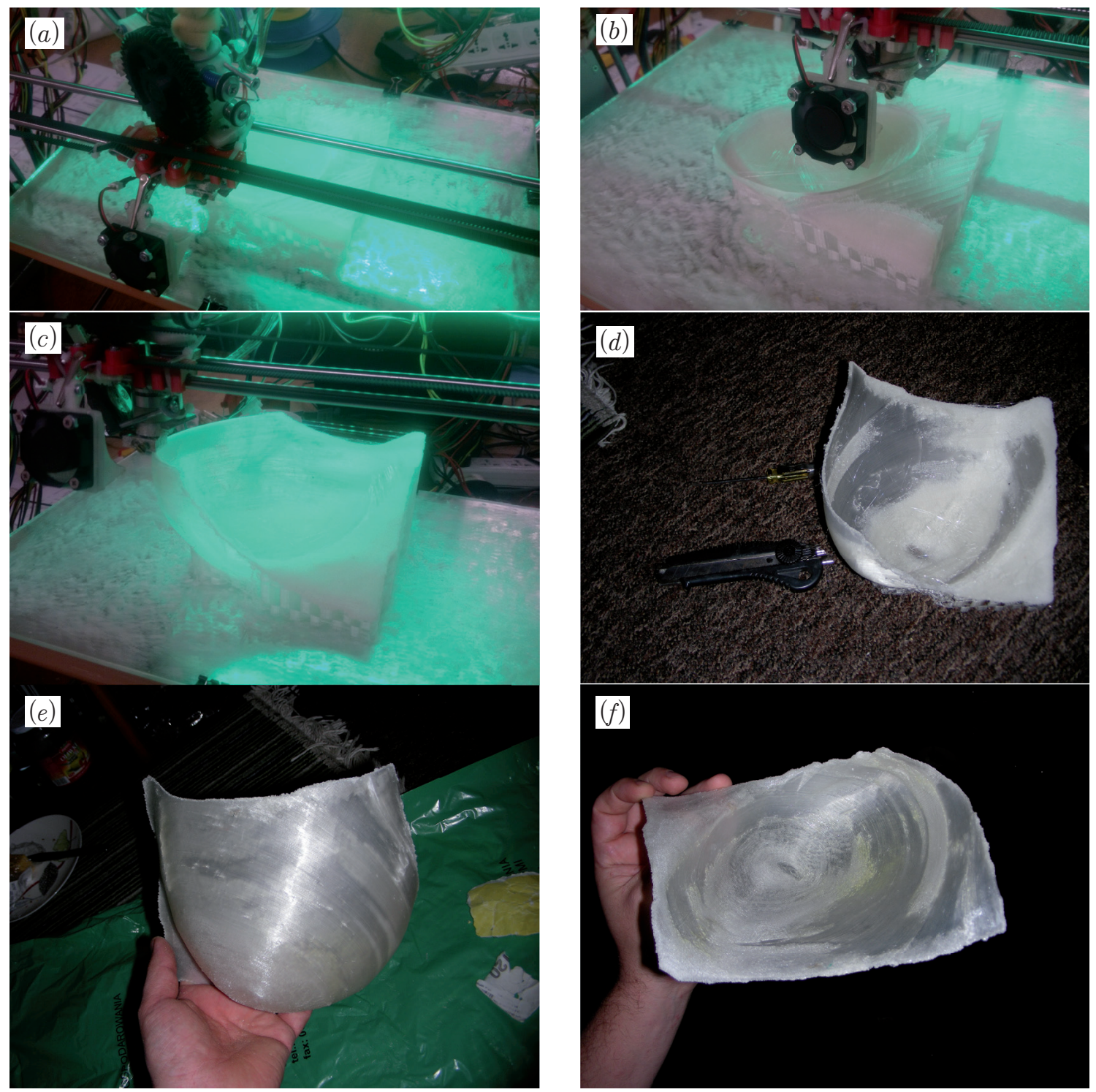

Fig. 4: Stage ll of printing of breast based on its 3D scan: data processing [4-7].

\section{$\checkmark$ shortened hospitalization,}

$\checkmark$ lack of additional corrective procedures,

$\checkmark$ lower cost,

$\checkmark$ increased patient's health- related quality of life (HRQoL) $[4-8,10]$.

Lack of standardization/unification in the area of 3D organs reconstruction makes every solution unique, and compartmental studies are very difficult. Reverse engineering technique to produce a new breast replica cast (NBRC) was developed by Ahcan et al. Preoperative laser 3D images were used to produce a mould of the contralateral healthy breast. The mould was used to help shape the new breast during surgical intervention. Such approach helps improve breast symmetry in terms of volume, position on the chast wall, projection, and contour [1]. Similar positive results of unilateral breast reconstructions using deep inferior epigastric artery perforator (DIEP) were reported by Tomita et al. What is more such intervention may be immediate or delayed. Cosmetic outcomes may be assessed/confirmed by the postoperative 3D measurements of bilateral breasts [10]. Reece et al. proposed 3D imaging of breasts in tilted position 
insted of traditional 3D imaging with the patient in an upright position. Aforementioned solutions may help describe changes in breast morphology when the patient is tilted to various angles [11]. Chhaya et al. investigated adipose tissue engineering: patient-tailored breast shaped scaffolds were fabricated from poly(d,I)-lactide polymer (fused deposition modelling - FDM) with pore sizes $>1 \mathrm{~mm}$ with geometry modelled in silico via 3D scanning. Afeention scaffolds were next:

$\checkmark$ seeded with human umbilical cord perivascular cells,

$\checkmark$ cultured under static conditions for $r$ weeks and subsequently two weeks in a biaxial rotating bioreactor,

$\checkmark$ seeded with human umbilical vein endothelial cells,

$\checkmark$ implanted subcutaneously into athymic nude rats for 24 weeks.

Angiogenesis resued a functional capillary network. Adipose tissue increased to $81.2 \%$ of overall tissue area at week 24 [12]. Complex individualized implants may significantly improve patient outcomes, influencing quicker development of reconstruction of traumatic injuries, facial and limbs prostheses, biologic, synthetic and hybrid implants [8].

Health care is most often limited in remote and resource-limited locations, usually overcomed by proposed 3D technologies. But 3D technologies also have severe limitations:

$\checkmark$ errors between estimated and real tissue volume since 3D surface imaging does not take skin envelope thickness into consideration,

$\checkmark 5-10 \%$ postoperative reduction as swelling disappears [10].

Currently observed limitations of the proposed approach can be overcomed by subsequent research, including common interdisciplinary efforts of scientists and clinicians.

\subsection{Directions for further studies}

Main directions for further research are:

$\checkmark$ patient-tailored 3D printed models for surgical education,

$\checkmark$ anthropomorphic 3D physical breast phantoms for development, optimization, and evaluation of $x$-ray breast imaging systems,

$\checkmark$ efficiency and safety, including anti-allergenic, water resistant, non-fragile features,

$\checkmark$ bio-ink printed implants, their vascularization and innervation,

$\checkmark$ preclinical animal studies,

$\checkmark$ interaction between various materials used in implants: bio-ink, metal, ceramic, plastic, etc.,

$\checkmark$ awareness and education of medical staff and patients,

$\checkmark$ new models of patient preparation, intervention, rehabilitation and care in patients with $3 D$ printed implants,

$\checkmark$ technologies of image gathering and processing, file storage and exchange, and data safety,

$\checkmark$ business models of such services [4-7, 13, 14, 15].

\section{Conclusions}

Results of this project influence both scientists and clinicians since proposed solutions are better customized, cheaper, wider available, and fitted to the local market. Despite relative novelty of the proposed solution, its further development may lead to the novel family of patient-tailored solutions and optimized 3D-based technology useful in everyday clinical practice. 3D-printed patient-tailored assistive technologies and artificial organs are only first of the wide spectrum of possible applications - 3D scans may be a part of electronic heath record (EHR) or even biometric passport/ID card.

\section{References and Notes}

[1] Ahcan, U., Bracun, D., Zivec, K., Pavlic, R., Butala, P. (2012). The use of 3D laser imaging and a new breast replica cast as a method to optimize autologous breast reconstruction after mastectomy. Breast, vol. 21, no. 2, pp. 183-189.

[2] Chae, M. P., Rozen, W. M., Spychal, R. T., Hunter-Smith, D. J. (2016). Breast volumetric analysis for aesthetic planning in breast reconstruction: a literature review of techniques. Gland Surgery, vol. 5, no. 2, pp. 212-226.

[3] Mehta, S., Byrne, N., Karunanithy, N., Farhadi, J. (2016). 3D printing provides unrivalled bespoke teaching tools for autologous free flap breast reconstruction. Journal of Plastic, Reconstructive \& Aesthetic Surgery, vol. 69, no. 4, pp. 578580.

[4] Mikołajewska, E., Macko, M., Mikołajewski, D., Ziarnecki, Ł., Stańczak, S., Kawalec, P. (2016). Medical and military applications of 3d printing. Zeszyty Naukowe Wyższa Szkoła Oficerska Wojsk Lądowych im. generała Tadeusza Kościuszki, vol. 179, no. 1, pp. 128-141

[5] Macko, M., Mikołajewska, E., Szczepański, Z., Augustyńska, B., Mikołajewski, D. (2016). Repository of images for reverse engineering and medical simulation purposes. Medical and Biological Sciences, vol. 30, no. 3, pp. 23-29. 
[6] Macko, M., Szczepański, Z., Mikołajewski, D., Mikołajewska, E., Nowak, J., Listopadzki, S. (2016). The method of artificial organs fabrication based on reverse engineering in medicine. Proceedings of The III International Scientific Conference: Morpho-Biomechanical and Psycho-Physical Aspects of Youth Lifestyle in V4 Countries, Napierała P. M. et al. (eds.) Bydgoszcz: Institute of Physical Education, Kazimierz Wielki University in Bydgoszcz, p. 45

[7] Mikołajewska, E., Macko, M., Ziarnecki, Ł., Stańczak, S., Kawalec, P., Mikołajewski, D. (2014) 3D printing technologies in rehabilitation engineering. Journal of Health Sciences, vol. 4, no. 12, pp. 78-83.

[8] Bauermeister, A. J., Zuriarrain, A., Newman, M. I. (2016). Three-Dimensional Printing in Plastic and Reconstructive Surgery: A Systematic Review. Annals of Plastic Surgery, vol. 77, no. 5, pp. 569-576.

[9] Chae, M. P., Rozen, W. M., McMenamin, P. G., Findlay, M. W. Spychal, R. T., Hunter-Smith, D. J. (2015). Emerging Applications of Bedside 3D Printing in Plastic Surgery. Frontiers in Surgery, no. 2, p. 25.

[10] Tomita, K., Yano K., Hata, Y., Nishibayashi, A., Hosokawa, K. (2015). DIEP flap breast reconstruction using 3-dimensional surface imaging and a printed mold. Plastic and Reconstructive Surgery Global Open., vol. 3, no. 3, p. e316.

[11] Reece, G. P., Merchant, F., Andon, J., Khatam, H., Ravi-Chandar, K., Weston, J., Fingeret, M. C., Lane, C., Duncan, K., Markey, M. K. (2015). 3D surface imaging of the human female torso in upright to supine positions. Medical Engineering \& Physics, vol. 37, no. 4, pp. 375-383.

[12] Chhaya, M. P., Melchels, F. P., Holzapfel, B. M., Baldwin, J. G., Hutmacher, D. W. (2015). Sustained regeneration of highvolume adipose tissue for breast reconstruction using computer aided design and biomanufacturing. Biomaterials, no. 52, pp. 551-560.

[13] Kiarashi, N., Nolte, A. C., Sturgeon, G. M., Segars, W. P., Ghate, S. V., Nolte, L. W., Samei, E., Lo, J. Y. (2015). Development of realistic physical breast phantoms matched to virtual breast phantoms based on human subject data. Medical Physics, vol. 42, no. 7, pp. 4116-4126.

[14] Hutmacher, D. W. (2016). Quo Vadis Breast Tissue Engineering? EbioMedicine, no. 6, pp. 24-25

[15] Chhaya, M. P., Balmayor, E. R., Hutmacher, D. W., Schantz, J. T. (2016). Transformation of breast reconstruction via additive biomanufacturing. Scientific Reports, no. 6, p. 28030.

\section{Biographical notes}

Dariusz Mikołajewski, PhD. Eng., born 1972, graduated from Military University of Technology in Warsaw, and Institute of Biocybernetics and Biomedical Engineering of the Polish Academy of Sciences in Warsaw, Poland and five postgraduate studies: in digital telecommunication, computer networks, project management (various methodologies), management of R\&D projects, and MBA.. Engineer and scientist, specialist in IT, applications of IT in medical sciences, biocybernetics, and medical robotics. He works for two research centers: 1) Institute of Mechanics and Applied Computer Science, Kazimierz Wielki University in Bydgoszzz, 2) Neurocognitive Laboratory, Centre for Modern Interdisciplinary Technologies, Nicolaus Copernicus University in Torun. He is also member of the InteRDoCTor research team. Author of more than 150 articles and chapters in the area of medical IT, biocybernetics, and biomedical engineering.

Piotr Prokopowicz, PhD., born 1974, Assistant Professor in Institute of Mechanics and Applied Computer Science, Kazimierz Wielki University. Ph.D. in Computer science, Institute of Fundamental Technological Research of the Polish Academy of Sciences, Warszawa. In 2014-2016 Chair of Database Systems and Computational Intelligence Department in Institute of Mechanics and Applied Computer Science, Kazimierz Wielki University. Main area of interests are the computational intelligence methods and its applications with special focus on fuzzy sets an systems. Member of Polish Information Processing Society (PTI - Polskie Towarzystwo Informatyczne).

Zbigniew Szczepański, MSc., 1984, graduated from Kazimierz Wielki University (MSc in mechatronics), assistant in Institute of Mechanics and Applied Computer Science, Kazimierz Wielki University.

Joanna Nowak, MSc., 1986, graduated from Kazimierz Wielki University (MSc in mechatronics), assistant in Institute of Mechanics and Applied Computer Science, Kazimierz Wielki University.

prof. Marek Macko, PhD., DSc., 1963, Vice-Rector for Development and Cooperation at Kazimierz Wielki University. A member of the Bydgoszcz Scientific Society (now a Vice President) and the Association of Engineers and Technicians of Polish Mechanics - a member of the Management Board of the Bydgoszcz Branch and also the President of the Section of Machine Operation.

Emilia Mikołajewska, PhD., born 1974, completed her PhD Thesis entitled: 'Application of NDT-Bobath method in rehabilitation of patients after ischemic stroke' in 2007 at the Medical University in Poznan. She has also completed four-years postgraduate studies for governmental title 'specialist in physiotherapy', postrgduate studies in R\&D projects management, and many additonal courses in neurorehabilitation of children and adults: NDT-Bobath basic for children (EBTA recognized), Bobath-Baby for newborn and premature children (EBTA recognize), basic and advanced courses Bobath for adults (IBITA recognized), PNF course, Kinesiology Taping course, 7-module Cyriax course, geriatric rehabilitation, etc. Assistant Professor in Department of Physiotherapy, Ludwik Rydygier Collegium Medicum in Bydgoszzz, Nicolaus Copernicus University in Torun, Poland. She is also researcher in Neurocognitive Laboratory, Centre for Modern Interdisciplinary Technologies, Nicolaus Copernicus University in Torun and member of the InteRDoCTorresearch team. Author of 16 books, 55 book chapters, and 270 articles in the area of rehabilitation and physiotherapy, especially neurorehabilitation and neurological physiotherapy. Reviewer in 110 scientific journals (also member of 20 Editorial Boards), several book chapters, and many grant applications. She also teaches courses on neurorehabilitation and assistive technology and acts as supervisor of specialization candidates. Her research interests cover areas: neurorehabilitation of adult patients, including post-stroke, neurorehabilitation of children, increasing of patients' quality of life using: assistive devices, telemedicine, telerehabilitation, cybertherapy, rehabilitative robotics, applications of IT, Al and integrated systems in medicine, co-operation within therapeutic multidisciplinary team, evidence based medicine in clinical practice, biomedical engineering in rehabilitation. 